\title{
Éditorial: \\ Qu'est-ce que soigner de manière éthique?
}

Le droit pour un individu de participer aux prises de décision qui le concernent constitue la pierre angulaire des règles légales qui prévalent en matière de soins. Mais, comme l'illustre l'article de Sarah Shidler, cette pierre angulaire doit aussi composer avec d'autres règles qui viennent la moduler. Les repaires légaux sont donc nombreux et leur harmonisation pas toujours facile. De plus ce droit de participer présuppose que l'individu a, dans les faits, l'opportunité de participer. Or, comme l'illustre encore très bien l'article de Shidler, cette opportunité n'est pas d'emblée évidente lorsqu'il s'agit d'une personne âgée souffrant de maladie chronique et demeurant en institution. Pour cette catégorie de personnes, la participation se heurte très souvent à des problèmes de communication. Pouvoir exercer son droit de participer implique de pouvoir communiquer. La communication est un impératif; c'est un art qu'il faut cultiver.

Mais la communication n'étant pas toujours possible et même, lorsqu'elle l'est, la communication n'étant jamais parfaite, les règles de droit ne peuvent à elles seules nous garantir que nous agissons bien. Au-delà des règles de droit, bien agir implique que celui qui agit le fasse d'une manière éthique. La question est donc «qu'est-ce que soigner de manière éthique?».

Je propose de définir la chose ainsi: soigner de manière éthique, c'est soigner selon le souci de soi, le souci d'autrui et le souci de chacun. Être un bon soignant c'est, au sens éthique, avoir développer une manière d'agir habitée par ces trois soucis. Et il faut donner ici au mot souci le sens de «préoccupation qui nous absorbe» comme dans l'expression "avoir le souci du détaily. Avoir le souci du détail, c'est accorder de l'importance aux détails; de la même façon agir selon le souci de soi, le souci d'autrui et le souci de chacun c'est accorder de l'importance à soi, à autrui et à chacun.

L'expression "souci de soi» peut, à première vue, étonner. En effet «souci de soì semble avoir une résonance égoïste. Mais notre étonnement s'estompera lorsque l'on soulignera que le "soi" est ici l'opposé, non pas de l'autrui ou du chacun, mais du "moi». Le souci de soi n'est pas le souci de moi. Le "soi» fait référence à la partie raisonnable de nous-mêmes par opposition au "moi» qui fait référence à la partie de nous-mêmes dominée par nos désirs irréfléchis. Le souci de soi signale la capacité humaine de choisir, de préférer ceci ou cela, d'agir intentionnellement, d'introduire des changements dans le cours des choses, de commencer quelque chose dans le monde. Le souci de soi se nourrit de désir et de raison et cherche l'harmonie de leur influence mutuelle. 
Le souci d'autrui peut aussi s'appeler la sollicitude. La sollicitude est une spontanéité bienveillante à l'égard d'autrui. La bienveillance à légard d'autrui implique la reconnaissance de l'autre comme mon semblable, la reconnaissance de son égalité. Mais cela ne suffit pas. Le souci d'autrui implique aussi la mutualité, c'est-à-dire un mouvement vers l'autre. Et dans ce mouvement, il faut qu'il y ait du «donner» et du "recevoir» et que ce donner et ce recevoir soient réciproques. À première vue, il n'est pas évident qu'une relation de soins puisse avoir un aspect réciproque. En effet, en première analyse, celui qui est malade est celui qui reçoit et celui qui soigne est celui qui donne. Où donc y a-t-il réciprocité? En m'inspirant des propos du philosophe Paul Ricoeur, je répondrai qu'elle se trouve dans la souffrance même. Il y a dans la souffrance même de celui qui souffre un «donner». Ce "donner» n'est pas puisé dans sa puissance d'agir mais dans sa faiblesse même. De tels propos peuvent étonner. Et pourtant! Prenons exemple sur un autre phénomène, celui du don. De nombreuses études sociologiques démontrent que la plupart des gens qui font du bénévolat estiment qu'en donnant ainsi de leur temps, ils reçoivent plus qu'ils ne donnent. Le donneur d'organe dira souvent qu'il reçoit autant que celui à qui il donne. Le donneur est transformé par le don autant que peut l'être le receveur. Aussi unilatéral que puisse paraître un don, il y a toujours retour dans le don. L'exemple du don illustre bien cette dynamique de réciprocité propre au souci d'autrui. Il y a sollicitude lorsque l'on donne et que ce "donner» est aussi un «recevoir».

Le souci de chacun se distingue du souci d'autrui. L'autrui signalait l'autre dans le cadre d'une relation interpersonnelle. Le chacun signale plutôt l'autre dans sa présence au sein d'une communauté, communauté prise au sens d'une "structure du vivre ensemble». Le chacun c'est l'autre en tant que membre de cette structure. Cette structure du vivre ensemble, il faut la comprendre comme un système de partage, de répartition ou de distribution entre ses membres. Ce système de partage tient compte d'un partage égal. Bien sur, en regard des soins, il ne s'agit pas de donner à chacun une part égale des soins disponible mais de donner à chacun une part égale à ce dont il a besoin. Le partage égal dont il est question ici est donc celui d'une égalité de rapport entre chaque fois une personne et un besoin de soins. Cela n'implique pas qu'il nous faille combler tous les besoins. Des ressources limitées peuvent impliquer une distribution limitée mais qui respectera alors l'égalité de rapport entre chaque fois une personne et son besoin et cela autant de fois qu'il y aura une personne avec un besoin.

Soigner selon le souci de soi, le souci d'autrui et le souci de chacun, telle est la réponse que je propose à la question "qu'est-ce que soigner de manière éthique?». Et agir ainsi ce n'est rien d'autre qu'agir selon l'excellence. Mais cette excellence n'est qu'une image directrice, une chose vers laquelle on tend. Par rapport à chaque cas particulier, l'excellence demeure floue. Face au cas particulier que j'ai devant moi, où se situe au juste l'excellence? La 
réponse à cette question ne peut venir que du jugement pratique. Un jugement pratique est une opération complexe à multiples dimensions. Cela implique d'abord de saisir le particulier, de saisir ce que le cas a d'unique. Cela implique ensuite d'avoir acquis un certain savoir, le savoir de l'expérience au sens le plus large du mot, ce qui n'est pas limitatif à sa seule expérience personnelle. Puis cela implique une mise en rapport du particulier et de l'expérience, mise en rapport qui est jaugée, évaluée, soupesée pour qu'au terme de cette délibération, un choix soit fait. Ce choix est celui qui est retenu parce qu'il est jugé le meilleur en regard de ce qui est, ici et maintenant, la forme concrète de l'excellence. Ce choix n'est donc pas un choix strictement basé sur une préférence personnelle car il ne fait pas abstraction de ce qu'enseigne l'expérience. Dans un processus de jugement pratique, il y a une mise en rapport avec l'expérience, expérience qui comprend certes l'expérience personnelle mais aussi, par l'intermédiaire du dialogue, celle des autres. Celui qui a développé son jugement pratique c'est ce qu'Aristote appelait "un homme prudent," celui qui a développé tout autant sa capacité d'agir selon le souci de soi, le souci d'autrui et le souci de chacun que sa capacité de jugement pratique.

Soigner de manière éthique implique donc que l'agent soit devenu un "clinicien-prudent». C'est ce clinicien-prudent qui mérite le nom de "praticien». Et comment devient-on praticien? De deux façons. La première c'est par l'imitation d'un modèle. C'est un mode de formation qui est bien connu en médecine. C'est pas l'imitation d'un modèle que l'on apprend à devenir clinicien. C'est l'essentiel de la formation acquise lorsque l'étudiant qui a fini son cours de médecine devient "résident». La "résidence» signale la transformation du médecin en clinicien. Il est intéressant de remarquer qu'en français le mot «patron» désigne à la fois un professeur de clinique et un modèle d'après lequel on taille un vêtement. Un "patron" c'est un guide, un modèle et c'est en le suivant que l'on devient soi-même clinicien. Le mode d'apprentissage n'est pas différent en éthique. On dira alors que le praticien sert de "patron» et qu'en le suivant on devient soi-même praticien. Bien sûr dans les deux cas, on fait face aux mêmes difficultés: ce ne sont pas tous les "patron» qui sont de vrais cliniciens comme ce ne sont pas tous les "patrons" qui sont de vrais praticiens. C'est le risque inhérent à ce genre d'apprentissage.

La seconde façon de devenir praticien, c'est la prise de décision ellemême. Au fur et à mesure de notre pratique, on prend des décisions et on voit qu'il y en a de meilleures que d'autres. Au fil des ans, notre habilité au jugement pratique s'améliore. S'instaure ainsi un processus évolutif, une transformation dans le temps. Et notre habileté au jugement pratique s'améliorera d'autant plus que les décisions à prendre seront éprouvantes. Il est difficile de devenir praticien si nos décisions ne sont jamais éprouvantes. Il est plus difficile au dermatologue de devenir praticien (au sens de clinicien-prudent) qu'au cancérologue, tout simplement parce que ce dernier est plus susceptible d'être impliqué dans des décision éprouvantes. 
Il faut, pour devenir praticien, accepter de prendre des décisions difficiles. Il faut donc en avoir la volonté et aussi avoir la volonté, après coup, de regarder d'un oeil critique nos décisions. Voilà les deux façons qui nous permettent de devenir praticien. Cela ne s'enseigne pas; cela implique plutôt une progressive transormation des personnes.

Gilles Voyer 